\title{
Connected Cruise Control Design Using Probabilistic Model Checking
}

\author{
Jin I. Ge ${ }^{1}$, Gábor Orosz ${ }^{1}$, and Richard M. Murray ${ }^{2}$
}

\begin{abstract}
In this paper, we synthesize a robust connected cruise controller with performance guarantee using probabilistic model checking, for a vehicle that receives motion information from several vehicles ahead through wireless vehicle-tovehicle communication. We model the car-following dynamics of the preceding vehicles as Markov chains and synthesize the connected cruise controller as a Markov decision process. We show through simulations that such a design is robust against imperfections in communication.
\end{abstract}

\section{INTRODUCTION}

For over a century, automotive engineers have been designing better cars in order to provide people with higher levels of mobility. However, the road transportation system is often plagued by stop-and-go traffic jams that bottleneck transportation efficiency [21] and also adversely impact individual vehicle's performance. Thus, it is necessary to consider new vehicle control strategies so that individual cars are able to 'steer' the traffic flow as a multi-agent system towards more desired states.

One primary factor under consideration is the longitudinal control of vehicle motion. Human drivers often perform poorly as vehicle controllers due to their relatively large reaction time and limited perception abilities. In particular, while most human drivers are able to maintain plant stability of a car (i.e., maintain a chosen speed when there are no external perturbations), they cannot guarantee string stability (cannot attenuate velocity fluctuations that travel backward along the vehicle chain). Using adaptive cruise control, one may improve string stability due to faster and more accurate sensing abilities and more sophisticated control strategies [4], [12]. However, adaptive cruise control cannot overcome the limitation that only motion information of the vehicle immediately ahead can be monitored by range sensors. This restricts the performance of the cruise controller and limits our ability to improve the traffic flow.

Therefore, researchers proposed to control vehicles using traffic flow information over a longer spatial horizon by utilizing vehicle-to-vehicle communication. Strategies include cooperative adaptive cruise control [3], [5], [13], [17]-[19], [22]-[24], [26] and connected cruise control [1], [6], [15], [16], [20], [25]. While cooperative adaptive cruise control is designed for platoons of vehicles with different levels of cooperation, connected cruise control simply utilizes motion

\footnotetext{
*This work is supported by NSF grant 1351456 .

1 Jin I. Ge and Gábor Orosz are with the Department of Mechanical Engineering, University of Michigan, Ann Arbor, MI 48105, USA gejin@umich.edu, orosz@umich.edu

2 Richard M. Murray is with Control and Dynamical Systems, California Institute of Technology, Pasadena, CA 91125, USA murray@cds.caltech.edu
}

information from multiple vehicles ahead, and does not require the surrounding vehicles to be cooperative. Then, by exploiting the received signals, the vehicle equipped with connected cruise controller may optimize its own performance [7], [8]. In this paper we include stochastic disturbances and introduce a new design method so that the controller can perform well in more realistic traffic scenarios. In particular, we take into account stochastic behavior of neighboring vehicles and include them in the controller synthesis. We write the connected vehicle system as a Markov decision process with stochastic disturbances, so that we can synthesize connected cruise controllers using probabilistic model checking (even when packet losses occur).

The layout of this paper is as follows. In Section II we introduce the human car-following model and a class of connected cruise controllers with similar structure. In Section III we formulate the optimal connected cruise control design problem in terms of probabilistic model checking, and demonstrate how stochastic velocity disturbances can be included in this framework. In Section IV we synthesize connected cruise controllers in a simple scenario and test their performance using numerical simulations. Finally, we conclude our findings in Section V.

\section{MODELING CONNECTED VEHICLE SYSTEMS}

In this section we model connected vehicle systems in discrete time under stochastic disturbances. We first describe the human car-following behavior using the optimal velocity model, and then set up a class of connected cruise controllers which are structurally similar to the optimal velocity model. The predefined structure specifies certain dynamic properties a synthesized controller should exhibit, and thus reduces the emphasis on well-formulated specifications in the type of linear temporal logic [10]. This setup may also give us more insights to the dynamics of the connected vehicle system and may scale better for connected vehicle systems consisting of a large number of vehicles.

\section{A. Human car-following model}

Here we discuss the human car-following behavior in nonemergency situations; see Fig. 1(a). While many human carfollowing models exist [14], the optimal velocity model has very simple mathematical form and provides great physical intuition. Thus, we choose it as a basis for the structure of our connected cruise controllers.

Based on [9], [15], [16], the optimal velocity model for the human-driven vehicle $i$ is

$$
\begin{aligned}
\dot{h}_{i}(t) & =v_{i+1}(t)-v_{i}(t), \\
\dot{v}_{i}(t) & =\alpha_{i}\left(V\left(h_{i}(t)\right)-v_{i}(t)\right)+\beta_{i}\left(v_{i+1}(t)-v_{i}(t)\right) .
\end{aligned}
$$




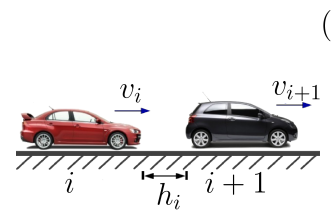

(a)

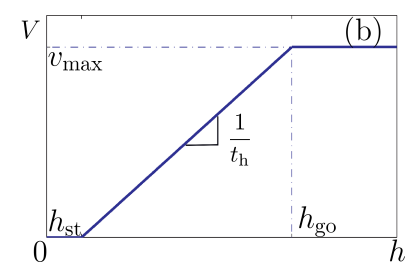

Fig. 1. (a): Single-lane car-following of human-driven vehicles showing the headway and the velocities. (b): The range policy (2) where $v_{\max }$ is the maximum allowed velocity, $h_{\mathrm{st}}$ is the smallest headway before the vehicle intends to stop, and $h_{\text {go }}$ is the largest headway after which the vehicle intends to maintain $v_{\max }$.

Here the dot stands for differentiation with respect to time $t$, $h_{i}$ denotes the headway, (i.e., the bumper-to-bumper distance between the vehicle $i$ and its predecessor), and $v_{i}$ denotes the velocity of vehicle $i$; see Fig. 1(a). According to (1) the acceleration is determined by two terms: the difference between the headway-dependent desired velocity and the actual velocity and the velocity difference between the vehicle and its predecessor. The gains $\alpha_{i}$ and $\beta_{i}$ are used to correct velocity errors. We neglect the human reaction time for simplicity.

The desired velocity is determined by the nonlinear range policy function

$$
V(h)=\left\{\begin{array}{lll}
0 & \text { if } \quad h \leq h_{\mathrm{st}}, \\
v_{\max } \frac{h-h_{\mathrm{st}}}{h_{\mathrm{go}}-h_{\mathrm{st}}} & \text { if } \quad h_{\mathrm{st}}<h<h_{\mathrm{go}}, \\
v_{\max } & \text { if } \quad h \geq h_{\mathrm{go}},
\end{array}\right.
$$

shown in Fig. 1(b). That is, the desired velocity is zero for small headways ( $h \leq h_{\mathrm{st}}$ ) and equal to the maximum speed $v_{\max }$ for large headways $\left(h \geq h_{\mathrm{go}}\right)$. Between these, the desired velocity increases with the headway linearly, which corresponds to the constant time headway $t_{\mathrm{h}}=$ $\left(h_{\mathrm{go}}-h_{\mathrm{st}}\right) / v_{\max }$. Many other range policies may be chosen, but the qualitative dynamics remain similar if the above characteristics are kept [16].

Note that (2) defines the steady-state behavior of vehicle $i$ and, in aggregation, the uniform traffic flow where vehicles travel with the same constant velocity and maintain the same constant headway:

$$
h_{i}(t) \equiv h^{*}, \quad v_{i}(t) \equiv v^{*}=V\left(h^{*}\right) .
$$

In a vehicle string, the equilibrium velocity $v^{*}$ is determined by the head vehicle while the equilibrium headway $h^{*}$ can be calculated from the range policy (2).

In this paper we use $v_{\max }=30[\mathrm{~m} / \mathrm{s}], h_{\mathrm{st}}=5[\mathrm{~m}], h_{\mathrm{go}}=$ $35[\mathrm{~m}]$ that corresponds to realistic traffic data [16], which results in the constant slope $V^{\prime}\left(h^{*}\right)=1[1 / \mathrm{s}]$ corresponding to the constant time headway $t_{\mathrm{h}}=1 / V^{\prime}\left(h^{*}\right)=1[\mathrm{~s}]$ in the region where $h_{\mathrm{st}}<h^{*}<h_{\mathrm{go}}, 0<v^{*}<v_{\max }$. In particular, we consider the system in the vicinity of the equilibrium $\left(h^{*}, v^{*}\right)=(20[\mathrm{~m}], 15[\mathrm{~m} / \mathrm{s}])$.

\section{B. Structured connected cruise controller}

We now consider the configuration shown in Fig. 2 where the vehicle at the tail is equipped with connected cruise

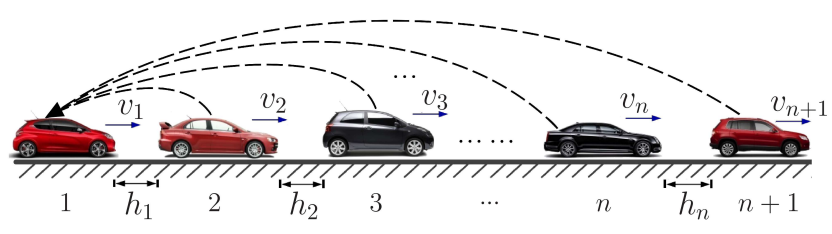

Fig. 2. A string of $n+1$ vehicles on a single lane. The CCC vehicle at the tail receives signals from human-driven vehicles ahead via vehicle-tovehicle communication. Dashed arrows indicate the flow of information in this connected vehicle system.

control and it receives motion information from the $n$ vehicles ahead through vehicle-to-vehicle communication (see dashed arrows terminating at vehicle 1). For simplicity, we assume that preceding vehicles are human-driven and can be described by (1).

Based on the dynamics of human-driven vehicles (1), we construct the connected cruise controller in the form

$$
\begin{aligned}
\dot{h}_{1}(t) & =v_{2}(t)-v_{1}(t), \\
\dot{v}_{1}(t) & =\alpha_{11}\left(V\left(h_{1}(t)\right)-v_{1}(t)\right)+\beta_{11}\left(w_{2}(t)-v_{1}(t)\right) \\
& +\sum_{i=2}^{n} \beta_{1 i}\left(w_{i+1}(t)-w_{i}(t)\right),
\end{aligned}
$$

where $\alpha_{11}$ and $\beta_{1 i}, i=1, \cdots, n$ are the feedback gains to be designed, and $w_{i}(t), i=2, \ldots, n+1$, are the velocity signals received by the connected cruise controller. We assume the headway $h_{1}$ and velocity $v_{1}$ of vehicle 1 are measured onboard, and thus are available to the controller. The received velocity signal $w_{i}(t)$ is equivalent to the actual velocity $v_{i}(t)$ without packet loss, but may differ when packet drops occur. Still, even for $w_{i} \neq v_{i}$ the equilibrium of the vehicle equipped with connected cruise control is given by (3).

In [7] we have shown that for a fixed group of preceding vehicles without packet loss, the optimal connected cruise control design gives $\beta_{1 i}$ that are constant in time but decrease with $i$. We can exploit this result later when synthesizing the optimal gains $\alpha_{11}$ and $\beta_{1 i}, i=1, \ldots, n$.

\section{Implementing connected cruise control}

Here we consider the human car-following model and the connected cruise control $(1,2)$ in the linear region and write them in discrete time to take into consideration the effect of sampling and zero-order hold in digital controllers. For simplicity we assume that the clocks used by the vehicles are synchronized.

We assume the dynamics of human-driven vehicles fluctuate around an equilibrium state (3), then we would like the dynamics of the vehicle equipped with connected cruise control $(1,2)$ to be in the vicinity of that state as well. Because $v^{*}=0$ describes the jammed state and $v^{*}=$ $v_{\max }$ corresponds to free flow, we focus on the equilibrium states where the desired velocity increases with the headway linearly (i.e., $h_{\mathrm{st}}<h<h_{\mathrm{go}}, 0<v<v_{\max }$, see the middle part in Fig. 1(b)).

We define the headway perturbations $\tilde{h}_{i}(t)=h_{i}(t)-h^{*}$ and velocity perturbations $\tilde{v}_{i}(t)=v_{i}(t)-v^{*}$ and linearize 
(1) about the equilibrium (3):

$$
\begin{aligned}
\dot{\tilde{h}}_{i}(t) & =\tilde{v}_{i+1}(t)-\tilde{v}_{i}(t), \\
\dot{\tilde{v}}_{i}(t) & =\frac{\alpha_{i}}{t_{\mathrm{h}}} \tilde{h}_{i}(t)-\left(\alpha_{i}+\beta_{i}\right) \tilde{v}_{i}(t)+\beta_{i} \tilde{v}_{i+1}(t) .
\end{aligned}
$$

Similarly we linearize the connected cruise controller (4)

$$
\begin{aligned}
\dot{\tilde{v}}_{1}(t) & =\alpha_{11}\left(\frac{1}{t_{\mathrm{h}}} \tilde{h}_{1}(t)-\tilde{v}_{1}(t)\right)+\beta_{11}\left(\tilde{w}_{2}(t)-\tilde{v}_{1}(t)\right) \\
& +\sum_{i=2}^{n} \beta_{1 i}\left(\tilde{w}_{i+1}(t)-\tilde{w}_{i}(t)\right),
\end{aligned}
$$

where $\tilde{w}_{i}(t)=w_{i}(t)-v^{*}$.

When using vehicle-to-vehicle communication the humandriven vehicles transmit their kinematic data intermittently in every $\Delta t$. To represent this sampling we first discretize (5) with time step $\Delta t$ :

$$
\begin{aligned}
\tilde{h}_{i}[k+1] & =\tilde{h}_{i}[k]+\Delta t\left(\tilde{v}_{i+1}[k]-\tilde{v}_{i}[k]\right), \\
\tilde{v}_{i}[k+1] & =\tilde{v}_{i}[k]+\Delta t \alpha_{i}\left(\frac{1}{t_{\mathrm{h}}} \tilde{h}_{i}[k]-\tilde{v}_{i}[k]\right) \\
& +\Delta t \beta_{i}\left(\tilde{v}_{i+1}[k]-\tilde{v}_{i}[k]\right),
\end{aligned}
$$

which approximates the sampled dynamics of human-driven vehicle $i$. Here we introduced the notation $\tilde{h}_{i}[k]=\tilde{h}_{i}(k \Delta t)$, $\tilde{v}_{i}[k]=\tilde{v}_{i}(k \Delta t)$.

We then discretize (6) with the same time step in order to describe the dynamics of the vehicle equipped with digital connected cruise control. We assume the digital controller uses zero-order hold by utilizing $\tilde{h}_{i}[k]$ and $\tilde{v}_{i}[k]$ in the time interval $[k \Delta t,(k+1) \Delta t)$, but for simplicity we ignore the $\mathcal{O}\left(\Delta t^{2}\right)$ terms. In this case, the discretized dynamics of the connected cruise controller is given by

$$
\begin{aligned}
\tilde{h}_{1}[k+1] & =\tilde{h}_{1}[k]+\Delta t\left(\tilde{v}_{2}[k]-\tilde{v}_{1}[k]\right), \\
\tilde{v}_{1}[k+1] & =\tilde{v}_{1}[k]+\Delta t \alpha_{11}\left(\frac{1}{t_{\mathrm{h}}} \tilde{h}_{1}[k]-\tilde{v}_{1}[k]\right) \\
& +\Delta t \beta_{11}\left(\tilde{w}_{2}[k]-\tilde{v}_{1}[k]\right) \\
& +\sum_{i=2}^{n} \Delta t \beta_{1 i}\left(\tilde{w}_{i+1}[k]-\tilde{w}_{i}[k]\right),
\end{aligned}
$$

Based on $(7,8)$, we formulate the controller synthesis problem in the next section.

\section{CONTROLler Synthesis USING PROBabilistic Model Checking}

In this section we present the framework that uses probabilistic model checking to synthesize connected cruise controllers considering stochastic events. We express the dynamics of the connected vehicle system $(7,8)$ as a Markov decision process and formulate the optimization objective accordingly. In particular, we consider stochastic velocity disturbance from the leading vehicle and also model the packet losses.

\section{A. Markov chain for human-driven vehicles}

Here we rewrite the sampled dynamics (7) of a humandriven vehicle as a Markov chain. We first quantize a bounded region of the state space into a finite number of cells. We consider bounded headway and velocity disturbances

$$
\left[\begin{array}{l}
\tilde{h}_{\min } \\
\tilde{v}_{\min }
\end{array}\right] \leq\left[\begin{array}{l}
\tilde{h} \\
\tilde{v}
\end{array}\right] \leq\left[\begin{array}{l}
\tilde{h}_{\max } \\
\tilde{v}_{\max }
\end{array}\right], \quad \tilde{v}_{\min } \leq \tilde{w}_{i} \leq \tilde{v}_{\max },
$$

for $i=2, \ldots, n$. With quantization sizes

$$
\Delta h=\frac{\tilde{h}_{\max }-\tilde{h}_{\min }}{N_{\mathrm{h}}}, \quad \Delta v=\frac{\tilde{v}_{\max }-\tilde{v}_{\min }}{N_{\mathrm{v}}},
$$

we define the quantized states and disturbances

$$
\begin{aligned}
x_{i} & =\text { floor }\left(\tilde{h}_{i} / \Delta h\right), \\
y_{i} & =\text { floor }\left(\tilde{w}_{i} / \Delta v\right), \\
z_{i} & =\text { floor }\left(\tilde{v}_{i} / \Delta v\right),
\end{aligned}
$$

for $i=2, \ldots, n$, with $x_{i}$ evaluated among $N_{\mathrm{h}}$ cells, and $y_{i}$ and $z_{i}$ in the $N_{\mathrm{v}}$ cells.

When considering a connected vehicle system of $n+1$ vehicles with stochastic velocity disturbance from the leading vehicle, we assume that $\tilde{v}_{n+1}$ is bounded and the probability transition matrix $\mathcal{C}_{n+1}$ of the stochastic signal $\tilde{v}_{n+1}[k]$ is known, that is,

$$
\mathbf{P}_{\mathrm{v}_{n+1}}[k+1]=\mathcal{C}_{n+1} \mathbf{P}_{\mathrm{v}_{n+1}}[k],
$$

where $\mathbf{P}_{\mathrm{v}_{n+1}}[k]$ is the probability distribution of the quantized state $z_{n+1}=$ floor $\left(\tilde{v}_{n+1} / \Delta v\right)$ at time step $k$.

Then, based on the discretized car-following model (7) and (12), we are able to write the dynamics of human-driven vehicles (7) as a Markov chain

$$
\mathbf{P}_{\text {human }}[k+1]=\mathcal{C}_{\text {human }} \mathbf{P}_{\text {human }}[k],
$$

where $\mathbf{P}_{\text {human }}[k]$ denotes the probability distribution of the quantized state $\left[\begin{array}{llllll}x_{2} & z_{2} & \ldots & x_{n} & z_{n} & z_{n+1}\end{array}\right]^{\mathrm{T}}$ at time step $k$, cf. (11).

We remark that (13) can be extended to include other stochastic events in traffic flow such as lane-changing.

\section{B. Markov decision process for connected cruise control}

We consider a finite set of feedback gains for the discretized linear connected cruise controller (8)

$$
\begin{aligned}
\alpha_{11} & =K_{\mathrm{a}} \Delta \alpha, \\
\beta_{1 i} & =K_{\mathrm{b} i} \Delta \beta,
\end{aligned}
$$

where $K_{\mathrm{a}}, K_{\mathrm{b} i} \in\{0, \ldots, m\}, m \in \mathbb{N}^{+}$, and $i=1, \ldots, n$. Now we are able to write the dynamics of the vehicle equipped with connected cruise controller as a Markov decision process

$\mathbf{P}_{1}[k+1]=\mathcal{C}_{\mathrm{CCC}}\left(z_{2}, y_{2}, \ldots, y_{n+1} ; K_{\mathrm{a}}, K_{\mathrm{b} 1}, \ldots, K_{\mathrm{b} n}\right) \mathbf{P}_{1}[k]$,

where $\mathbf{P}_{1}[k]$ is the probability distribution of the state $\left[\begin{array}{ll}x_{1} & z_{1}\end{array}\right]^{\mathrm{T}}$, while the probability transition matrix $\mathcal{C}_{\mathrm{CCC}}$ depends on the actual velocity disturbance $z_{2}$, the received velocity signals $y_{i}$ for $i=2, \ldots, n+1$, and undetermined feedback gains $K_{\mathrm{a}}, K_{\mathrm{b} i}$ for $i=2, \ldots, n$, at time step $k$. 
Now we specify the relation between the received signal $w_{i}$ and the actual velocity $v_{i}$ in order to complete the description of the connected vehicle system. When there is no packet drop, the received signal corresponds to the actual motion of vehicle $i$

$$
w_{i}[k]=v_{i}[k] \quad \Rightarrow \quad y_{i}[k]=z_{i}[k],
$$

for $i=2, \ldots, n$, cf. (11). However, according to the vehicle-to-vehicle communication protocol, when a packet is dropped, it will not be resent at the next transmission, and the connected cruise controller uses the information received in the previous time step. Thus, we have

$$
w_{i}[k]=v_{i}[k-1] \quad \Rightarrow \quad y_{i}[k]=z_{i}[k-1] .
$$

In [2] it was found that the probability of more than one consecutive packet drops is relatively low. Thus we only consider non-consecutive packet drops.

Therefore the dynamics of the connected vehicle system can be described by the Markov decision process $(13,15,16)$ when there is no packet drop at time step $k$, while $(13,15,17)$ is used when there is packet drop at time step $k$. The dynamics of packet drop can be integrated into the setup, but in this paper we only consider the scenario where every second packet is lost.

In order to synthesize the controller we set up the following stochastic optimization problem:

$$
\begin{aligned}
& \min \sum_{k=1}^{N} \mathbb{E}\left(\gamma_{\mathrm{h}}\left(x_{1}[k]\right)^{2}+\gamma_{\mathrm{v}}\left(z_{1}[k]\right)^{2}+\left(u_{1}[k]\right)^{2}\right), \\
& {\left[\begin{array}{c}
x_{1}[N] \\
z_{1}[N]
\end{array}\right] \in \boldsymbol{\Omega}_{\mathrm{end}}, \quad\left[\begin{array}{c}
x_{1}[k] \\
z_{1}[k]
\end{array}\right] \in \boldsymbol{\Omega}_{\mathrm{safe}},}
\end{aligned}
$$

for $k=1, \ldots, N$, where $\mathbb{E}$ denotes the expected value, $\gamma_{\mathrm{h}}$ and $\gamma_{\mathrm{v}}$ are the weighting factors for the headway and velocity fluctuations, respectively, while $u_{1}$ is the acceleration of vehicle 1 defined in $(4,8,11)$. We also require the dynamics of the vehicle equipped with connected cruise control to stay within the safe region $\Omega_{\text {safe }}$ in the quantized state space and to reach the desired region $\Omega_{\text {end }}$ at the end of the optimization. Note that the time horizon $N$ is not given but depends on when the trajectory hits $\boldsymbol{\Omega}_{\text {end }}$.

The optimization problem $(13,15,16,17,18)$ can be solved using the stochastic model checking software PRISM [11]. The synthesized controller will be given as a map between the strategy $K_{\mathrm{a}}, K_{\mathrm{b} i}$ and the headway and velocity fluctuations:

$$
\begin{aligned}
& \alpha_{11}=K_{\mathrm{a}}\left(x_{1}, z_{1}, z_{2}, y_{2}, \ldots, y_{n+1}\right) \Delta \alpha, \\
& \beta_{1 i}=K_{\mathrm{b} i}\left(x_{1}, z_{1}, z_{2}, y_{2}, \ldots, y_{n+1}\right) \Delta \beta,
\end{aligned}
$$

for $i=1, \ldots, n$, cf. (14). Though such maps may not be deterministic for general stochastic optimization problems, for this particular problem we have a deterministic correspondence between the quantized states $\left(x_{1}, z_{1}, z_{2}, y_{2}, \ldots, y_{n+1}\right)$ and the optimized feedback gains at each time step.

Note that due to the optimization setup, no feedback gains are specified for $\left(x_{1}, z_{1}\right) \in \boldsymbol{\Omega}_{\mathrm{end}}$. One simple strategy in such cases is to set the feedback gains zero inside the end region, which we implement in this paper.

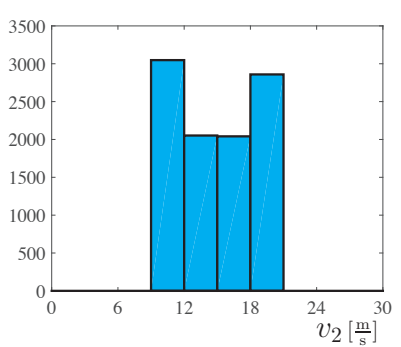

Fig. 3. Histogram of 10000 velocity profiles $v_{2}(t)$ evaluated at $t=50$ [s], where $v_{2}(t)$ is generated by $(12,24,25)$.
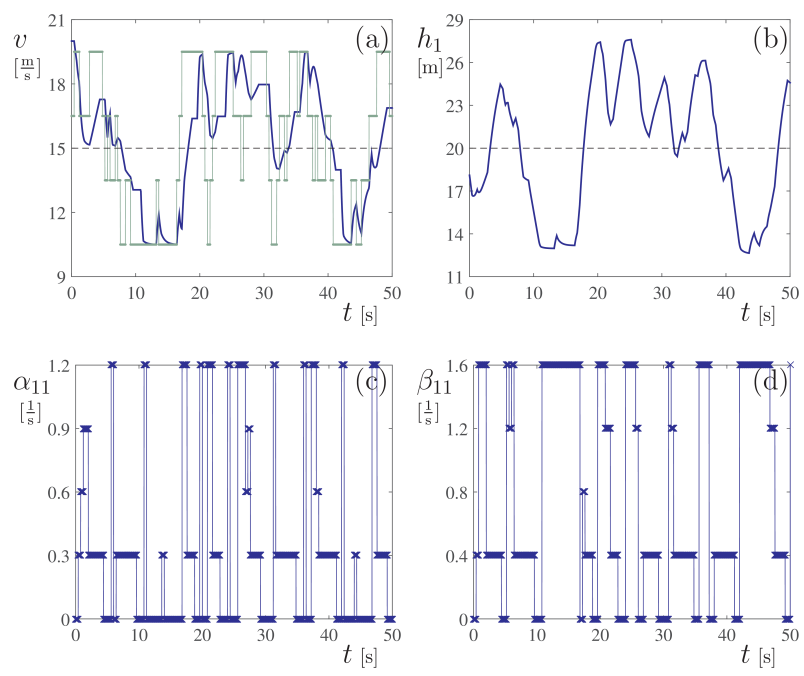

Fig. 4. (a,b): Headway and velocity responses for a $(1+1)$-vehicle string as functions of time. The blue solid curves show the headway $h_{1}(t)$ and the velocity $v_{1}(t)$ for the vehicle equipped with connected cruise control. The green dots show the velocity $v_{2}(t)$ of the leading vehicle, which is the same as the received velocity signal $w_{2}(t)$. (c,d): The feedback gains $\alpha_{11}$ and $\beta_{11}$ used by the controller.

\section{Simulation}

In this section, we demonstrate the simplest results of probabilistic model checking by designing connected cruise control for a two-vehicle system (i.e., $n=1$ in (4)). Here we only have two feedback gains:

$$
\begin{aligned}
& \alpha_{11}=K_{\mathrm{a}}\left(x_{1}, z_{1}, z_{2}, y_{2}\right) \Delta \alpha, \\
& \beta_{11}=K_{\mathrm{b} 1}\left(x_{1}, z_{1}, z_{2}, y_{2}\right) \Delta \beta .
\end{aligned}
$$

We first synthesize the connected cruise controller using $(15,16,17,18)$ in discrete time and quantized space, and then simulate the continuous-time controller (4) with zero-orderhold assumption on the disturbance and feedback gains.

We consider the time step $\Delta t=0.4[\mathrm{~s}]$ and the quantization size $\Delta h=3[\mathrm{~m}], \Delta v=3[\mathrm{~m} / \mathrm{s}]$, and we set the fluctuation bounds $\tilde{h}_{\max }=12[\mathrm{~m}], \tilde{h}_{\min }=-12[\mathrm{~m}]$, $\tilde{v}_{\max }=12[\mathrm{~m} / \mathrm{s}]$ and $\tilde{v}_{\min }=-12[\mathrm{~m} / \mathrm{s}]$. Thus, the number of headway and velocity cells are $N_{\mathrm{h}}=8$ and $N_{\mathrm{v}}=8$, respectively. For fluctuations with tighter bounds, the time step and quantization size can be proportionally reduced. We set the total number and increments for the quantized feedback gains as $m=5, \Delta \alpha=0.3[1 / \mathrm{s}], \Delta \beta=0.4[1 / \mathrm{s}]$. The weighting factors are chosen to be $\gamma_{\mathrm{h}}=1$ and $\gamma_{\mathrm{v}}=1$. 

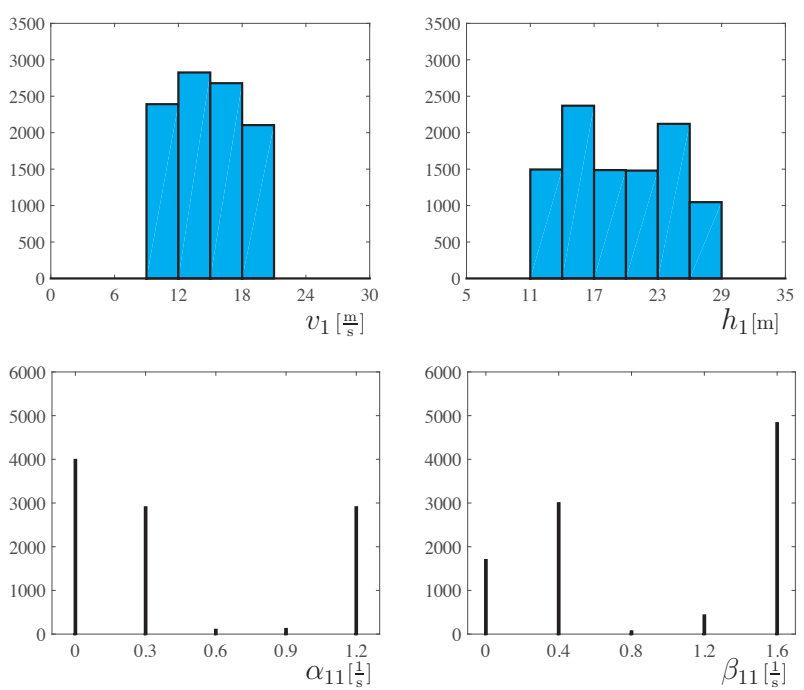

Fig. 5. (a,b): Histograms of velocity and headway at $t=50[\mathrm{~s}]$ in simulations of a $(1+1)$-vehicle string with stochastic velocity disturbance $(4,12,24,25)$. (c,d): The histogram of feedback gains $\alpha_{11}$ and $\beta_{11}$ used by the connected cruise controller at $t=50[\mathrm{~s}]$.

We define the safety region and the end condition as

$$
\begin{aligned}
& \boldsymbol{\Omega}_{\mathrm{safe}}=\left\{\left[\begin{array}{l}
x_{1} \\
z_{1}
\end{array}\right] \mid\left[\begin{array}{l}
1 \\
1
\end{array}\right] \leq\left[\begin{array}{l}
x_{1} \\
z_{1}
\end{array}\right] \leq\left[\begin{array}{l}
8 \\
8
\end{array}\right]\right\}, \\
& \boldsymbol{\Omega}_{\mathrm{end}}=\left\{\left[\begin{array}{l}
x_{1} \\
z_{1}
\end{array}\right] \mid\left[\begin{array}{l}
4 \\
4
\end{array}\right] \leq\left[\begin{array}{l}
x_{1} \\
z_{1}
\end{array}\right] \leq\left[\begin{array}{l}
5 \\
5
\end{array}\right]\right\} .
\end{aligned}
$$

We consider bounded fluctuation of velocity $v_{2}$

$$
v^{*}-2 \Delta v \leq v_{2} \leq v^{*}+2 \Delta v
$$

that yields

$$
3 \leq z_{2} \leq 6,
$$

and assume the probability transition matrix

$$
\mathcal{C}_{2}=\left[\begin{array}{cccc}
0.7840 & 0.3200 & 0 & 0 \\
0.2160 & 0.3800 & 0.3080 & 0 \\
0 & 0.3000 & 0.3840 & 0.2160 \\
0 & 0 & 0.3080 & 0.7840
\end{array}\right]
$$

cf. (12). This probability transition matrix has an eigenvalue at 1 and the eigenvector $\mathcal{K}=$ $\left[\begin{array}{llll}0.3058 & 0.2064 & 0.2011 & 0.2867\end{array}\right]^{\mathrm{T}}$ gives the steadystate probability distribution of $z_{2}$. As an illustration, we generate 10000 discrete-time velocity profiles $\tilde{v}_{2}[k]$ based on $(12,24)$, and correspondingly define the continuous-time velocity profiles

$$
v_{2}(t)=v^{*}+\tilde{v}_{2}[k], \quad t \in[k \Delta t,(k+1) \Delta t) .
$$

We sample each $v_{2}(t)$ at time $t=50$ [s], and plot the histogram in Fig. 3. One can observe that the histogram corresponds to the steady-state distribution $\mathcal{K}$.

\section{A. Controller synthesis with no packet loss}

Here we synthesize the optimal connected cruise controller for the two-vehicle system $(15,16,18)$ with no packet loss and test the nonlinear controller (4) with synthesized gains (19) in continuous time using zero-order hold.
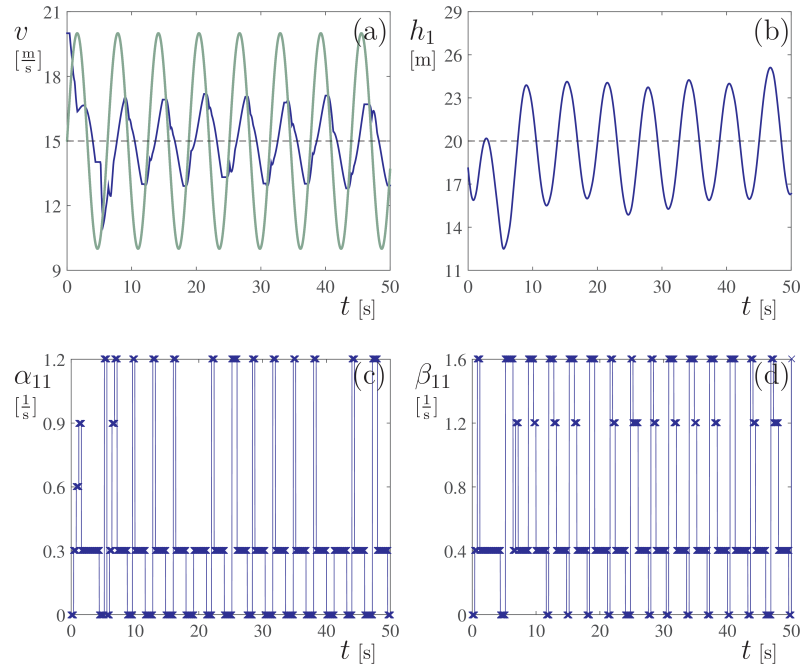

Fig. 6. (a,b): Velocity and headway responses for a $(1+1)$-vehicle string under sinusoidal disturbance. (c,d): The feedback gains $\alpha_{11}$ and $\beta_{11}$ used by the connected cruise controller. The notations are the same as in Fig. 4.

We first demonstrate the performance of the connected cruise controller under a random velocity disturbance generated by $(12,24,25)$. The headway and velocity responses and feedback gains of the vehicle equipped with connected cruise control are plotted in Fig. 4 as functions of time. We can see from Fig. 4(a) that the synthesized controller is able to follow the preceding vehicle's motion well, while maintaining reasonable headway as shown in Fig. 4(b). This simulation demonstrates that the connected cruise controller performs well under stochastic disturbances.

In order to evaluate the performance of the controller in the statistical sense, we repeat the simulation $(4,12,24,25)$ for 10000 different inputs, collect the value of headway, velocity, and feedback gains at $t=50[\mathrm{~s}]$ in each run, and plot the corresponding histograms in Fig. 5. By comparing Fig. 3 and Fig. 5(a) we find that the probability of $z_{1} \in\{4,5\}$ is larger than $z_{2} \in\{4,5\}$, which show that the controller performs well in disturbance rejection. On the other hand, Fig. 5(b) shows that the headway fluctuation $x_{1}$ is well contained within the safe region, cf. (21).

To further evaluate the disturbance-rejection performance of the synthesized controller, we consider a sinusoidal velocity profile

$$
v_{2}(t)=v^{*}+v^{\mathrm{amp}} \cos (\omega t)
$$

where $v^{\mathrm{amp}}=5[\mathrm{~m} / \mathrm{s}]$ and $\omega=1[1 / \mathrm{s}]$. The simulation results are shown in Fig. 6. In Fig. 6(a) we see that the velocity fluctuation of the vehicle equipped with connected cruise control (blue curve) has smaller amplitude than the preceding vehicle (green curve), and the headway fluctuations are also kept within safety region. Due to the periodicity of $v_{2}(t)$, the feedback gains $\alpha_{11}$ and $\beta_{11}$ also exhibit certain periodic nature. We note that while static gains are also able to suppress the velocity fluctuations [7], dynamic gains may provide us more flexibility under stochastic influences from the traffic. 

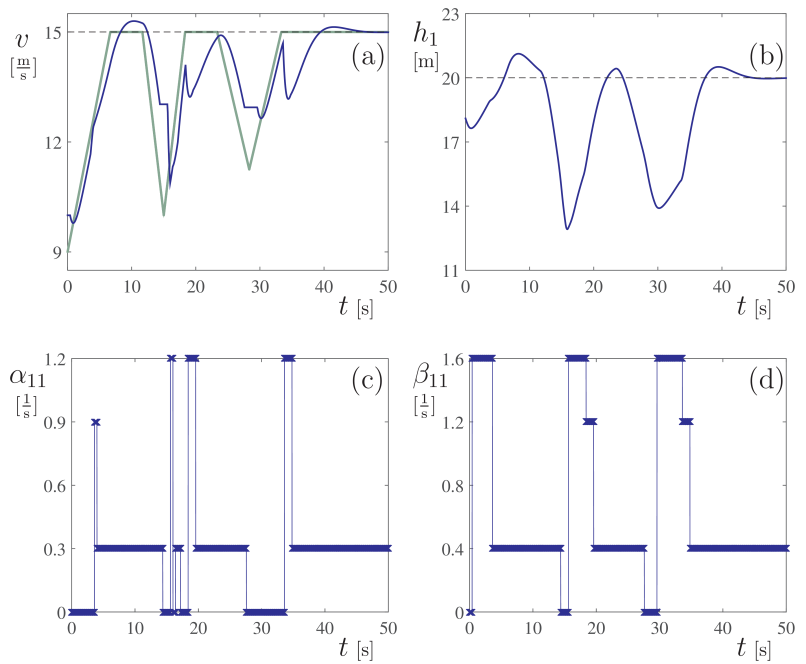

Fig. 7. (a,b): Velocity and headway responses for a $(1+1)$-vehicle string under triangular disturbance. (c,d): The feedback gains $\alpha_{11}$ and $\beta_{11}$ used by the connected cruise controller. The notations are the same as in Fig. 4.
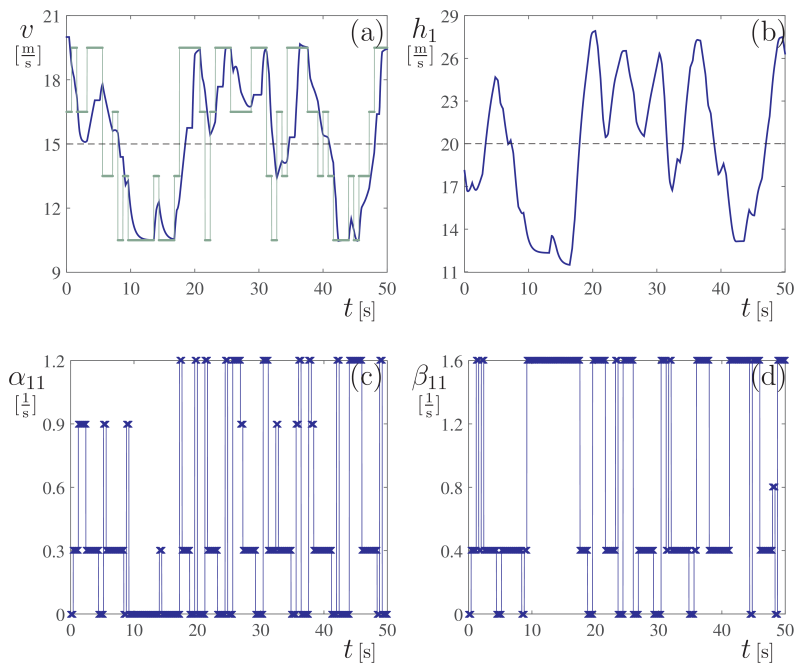

Fig. 8. (a,b): Velocity and headway responses for a $(1+1)$-vehicle string under packet loss. The blue solid curves show the headway $h_{1}$ and the velocity $v_{1}$ for the CCC vehicle. The green dots show the observed velocity $w_{2}$ of the leading vehicle 2. (c,d): The feedback gains $\alpha_{11}$ and $\beta_{11}$ used by the connected cruise controller.

Finally, we test the synthesized controller using a triangular velocity signal that is more commonly seen in traffic flow, and plot the results in Fig. 7. In this case, the leading vehicle starts with a velocity below the equilibrium $v^{*}$, and then accelerates to $v^{*}$ with constant acceleration, and after two dips its velocity settles down to $v^{*}$; see the green curve in Fig. 7(a). The controlled vehicle responds to the velocity fluctuations well, especially that $v_{1}$ (blue curve) has smaller local minima than $v_{2}$ (green curve), indicating successful attenuation of velocity perturbations. Moreover, the headway fluctuations are also kept within the safety region, see Fig. 7(b). Note that in Fig. 7(c,d) the feedback gains $\alpha_{11}$ and $\beta_{11}$ settle down to their respective non-zero minimal value as the states of the controlled vehicle converge to the equilibrium.
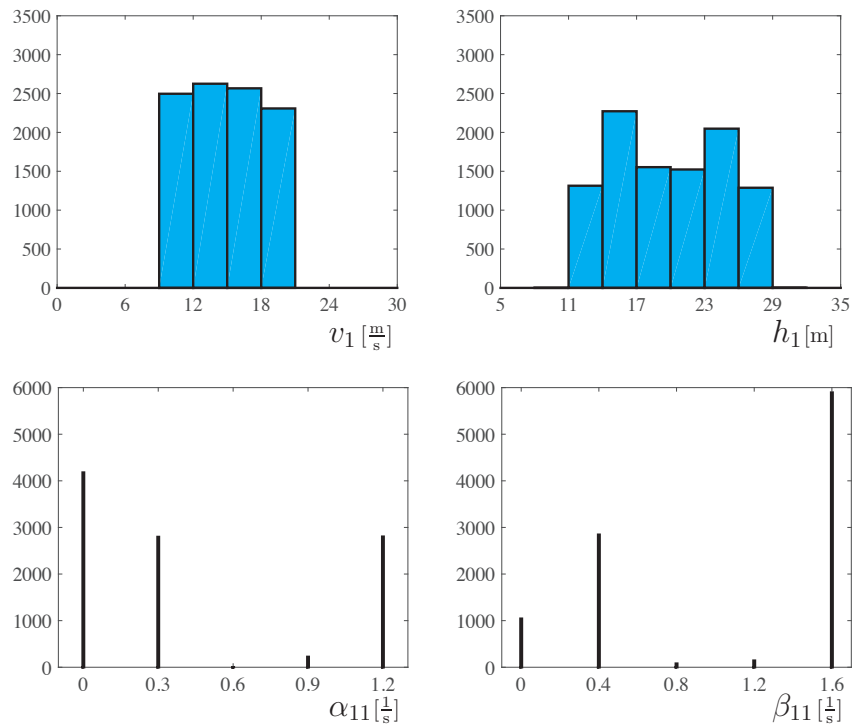

Fig. 9. (a,b): Histograms of velocity and headway at $t=50[\mathrm{~s}]$ in simulations of a $(1+1)$-vehicle string with stochastic velocity disturbance and packet losses. (c,d): Histogram of feedback gains $\alpha_{11}$ and $\beta_{11}$ used by the connected cruise controller at $t=50$ [s].

\section{B. Controller synthesis with packet loss}

Here we still consider a two-vehicle system but assume the connected cruise controller fails to receive every second packet of the velocity signal sent by vehicle 2 . We synthesize an optimal controller for $(15,16,17,18)$ similarly as in the previous subsection.

In Fig. 8 we test the synthesized controller using the same velocity signal $v_{2}$ as in Fig. 4(a), but in Fig. 8(a) the velocity profile $w_{2}$ received by vehicle 1 is plotted as green dots. We note that the dynamic gains in the no-packetloss and packet-loss cases are clearly distinctive from each other, cf. Fig. 4(c,d) and Fig. 8(c,d). This demonstrates that the synthesized controller is able to adapt to the changes due to packet losses. Moreover, the generated car-following dynamics is robust against the packet losses, as shown by the similarities of the blue curves in Fig. 4(a,b) and Fig. 8(a,b). In both cases, the controlled vehicle is able to follow the preceding vehicle while maintaining the desired time headway.

Similarly, to demonstrate the controller performance statistically, we perform 10000 runs and plot the histograms for the headway, velocity, and feedback gains at time $t=50$ [s] in Fig. 9. We see that with packet losses, the connected cruise controller still maintains similar distributions for the velocity and headway fluctuations as in Fig. 5. However, we note that there are considerable differences in the distribution of feedback gain $\beta_{11}$ between Fig. 5(d) and Fig. 9(d), and this demonstrates the necessity of considering packet losses in the system.

\section{CONCLUSIONS}

In this paper we demonstrated the design of connected cruise control using probabilistic model checking. Our method was based on stochastic optimal control and was able 
to accommodate stochastic events from the traffic flow and also react to packet drops in vehicle-to-vehicle communication. We showed through simulations that the synthesized controller is robust against imperfect communication and may be used in connected vehicle systems with a larger number of vehicles.

\section{ACKNOWLEDGMENT}

Jin Ge and Gábor Orosz thank Professor A. Galip Ulsoy for the very insightful discussions.

\section{REFERENCES}

[1] S. S. Avedisov and G. Orosz, "Nonlinear network modes in cyclic systems with applications to connected vehicles," Journal of Nonlinear Science, vol. 25, no. 4, pp. 1015-1049, 2015.

[2] F. Bai and H. Krishnan, "Reliability analysis of DSRC wireless communication for vehicle safety applications," in Intelligent Transportation Systems Conference, IEEE, 2006, pp. 355-362.

[3] E. Chan, P. Gilhead, P. Jelinek, P. Krejci, and T. Robinson, "Cooperative control of SARTRE automated platoon vehicles," in Proceedings of the 19th ITS World Congress, 2012.

[4] L. C. Davis, "Effect of adaptive cruise control systems on traffic flow," Physical Review E, vol. 69, no. 6, p. 066110, 2004.

[5] M. di Bernardo, A. Salvi, and S. Santini, "Distributed consensus strategy for platooning of vehicles in the presence of time varying heterogeneous communication delays," IEEE Transaction on Intelligent Transportation Systems, vol. 16, no. 1, pp. 102-112, 2015.

[6] J. I. Ge and G. Orosz, "Dynamics of connected vehicle systems with delayed acceleration feedback," Transportation Research Part C, vol. 46, pp. 46-64, 2014.

[7] —, "Optimal control of connected vehicle systems," in Proceedings of the 53rd IEEE Conference on Decision and Control. IEEE, 2014, pp. 4107-4112.

[8] _ - "Optimal control of connected vehicle systems with communication delay and driver reaction time," IEEE Transactions on Intelligent Transportation Systems, p. submitted, 2015.

[9] J. I. Ge, G. Orosz, D. Hajdu, T. Insperger, and J. Moehlis, "To delay or not to delay - stability of connected cruise control," in Time Delay Systems - Theory, Numerics, Applications and Experiments, Advances in Delays and Dynamics, T. Insperger, T. Ersal, and G. Orosz, Eds., vol. 7. Springer, 2016, p. accepted.

[10] D. Han, Y. Mo, and R. M. Murray, "Synthesis of distributed longitudinal control protocols for a platoon of autonomous vehicles," in Proceedings of the 54th IEEE Conference on Decision and Control. IEEE, 2015.

[11] M. Kwiatkowska, G. Norman, and D. Parker, "PRISM 4.0: Verification of probabilistic real-time systems," in Proceedings of the 23rd International Conference on Computer Aided Verification, ser. CAV'11. Springer, 2011, pp. 585-591.

[12] K. Li and P. Ioannou, "Modeling of traffic flow of automated vehicles," IEEE Transactions on Intelligent Transportation Systems, vol. 5, no. 2, pp. 99-113, 2004.

[13] V. Milanes, J. Alonso, L. Bouraoui, and J. Ploeg, "Cooperative maneuvering in close environments among cybercars and dual-mode cars," IEEE Transactions on Intelligent Transportation Systems, vol. 12, no. 1, pp. 15-24, 2011.

[14] K. Nagel, P. Wagner, and R. Woesler, "Still flowing: Approaches to traffic flow and traffic jam modeling," Operations Research, vol. 51, no. 5, pp. 681-710, 2003.

[15] G. Orosz, "Connected cruise control: modeling, delay effects, and nonlinear behavior," Vehicle System Dynamics, vol. 54, no. 8, pp. 1147-1176, 2016.

[16] G. Orosz, R. E. Wilson, and G. Stépán, "Traffic jams: dynamics and control," Philosophical Transactions of the Royal Society A, vol. 368, no. 1928, pp. 4455-4479, 2010.

[17] J. Ploeg, E. Semsar-Kazerooni, G. Lijster, N. van de Wouw, and H. Nijmeijer, "Graceful degradation of cooperative adaptive cruise control," IEEE Transactions on Intelligent Transportation Systems, vol. 16, no. 1, pp. 488-497, 2015.
[18] J. Ploeg, D. Shukla, N. van de Wouw, and H. Nijmeijer, "Controller synthesis for string stability of vehicle platoons," IEEE Transactions on Intelligent Transportation Systems, vol. 15, no. 2, pp. 845-865, 2014.

[19] J. Ploeg, N. van de Wouw, and H. Nijmeijer, " $\mathcal{L}_{\mathrm{p}}$ string stability of cascaded systems: application to vehicle platooning," IEEE Transactions on Control Systems Technology, vol. 22, no. 2, pp. 786-793, 2014.

[20] W. B. Qin, M. M. Gomez, and G. Orosz, "Stability and frequency response under stochastic communication delays with applications to connected cruise control design," IEEE Transactions on Intelligent Transportation Systems, p. appeared online, 2016.

[21] D. Schrank, B. Eisele, and T. Lomax, "2012 Annual Urban Mobility Report," Texas Transportation Institute, College Station, TX, Progressreport 70, 2012.

[22] B. van Arem, C. J. G. van Driel, and R. Visser, "The impact of cooperative adaptive cruise control on traffic-flow characteristics," IEEE Transactions on Intelligent Transportation Systems, vol. 7, no. 4, pp. 429-436, 2006.

[23] E. van Nunen, R. J. A. E. Kwakkernaat, J. Ploeg, and B. D. Netten, "Cooperative competition for future mobility," IEEE Transactions on Intelligent Transportation Systems, vol. 13, no. 3, pp. 1018-1025, 2012.

[24] M. Wang, W. Daamen, S. P. Hoogendoorn, and B. van Arem, "Rolling horizon control framework for driver assistance systems. part II: Cooperative sensing and cooperative control," Transportation Research Part C, vol. 40, pp. 290-311, 2014.

[25] L. Zhang and G. Orosz, "Motif-based analysis of connected vehicle systems: delay effects and stability," IEEE Transactions on Intelligent Transportation Systems, vol. 17, no. 6, pp. 1638-1651, 2016.

[26] Y. Zheng, S. E. Li, J. Wang, D. Cao, and K. Li, "Stability and scalability of homogeneous vehicular platoon: Study on the influence of information flow topologies," IEEE Transactions on Intelligent Transportation Systems, vol. 17, no. 1, pp. 14-26, 2016. 